\title{
A Case of Refractory Dysphagia in the Context of Polymyositis Treat- ed with Subcutaneous Immunoglobulin and Review of the Literature
}

\author{
Vacchi C*, Sebastiani M, Manfredi A and Ferri C \\ Rheumatology Unit, Azienda Policlinico di Modena, University of Modena and Reggio Emilia, Italy \\ *Corresponding author: Vacchi C, Rheumatology Unit, Azienda Policlinico di Modena, University of Modena and Reggio \\ Emilia, Italy, E-mail: vacchi.caterina@outlook.it
}

\begin{abstract}
Polymyositis (PM) is a systemic inflammatory disease affecting muscles and several other organs, including gastrointestinal system, in which the main symptom is dysphagia.

Here, we describe a case of a 57-years-old woman presenting a difficult-to-manage dysphagia related to polymyositis, treated with benefit with Subcutaneous Immunoglobulins (SClg). Beyond symptomatic therapies, immunosuppressive therapies (glucocorticoids, methotrexate, azathioprine, mycophenolatemofetil, and cyclosporine) are the main treatment of PM related dysphagia. Nowadays, few data are available to evaluate effectiveness and safety of intravenous immunoglobulins on esophageal involvement in refractory PM and only anecdotal cases are reported about the use of SClg in the treatment of dysphagia in inflammatory myopathies (including inclusion body myositis). Up to date, this is the second observation describing an improvement of dysphagia in a subject affected by PM treated with SClg; further studies are needed to evaluate the effectiveness of SClg in the management of these patients.
\end{abstract}

\section{Keywords}

Polymyositis, Dysphagia, Therapy, Subcutaneous immunoglobulins

\section{Introduction}

Polymyositis (PM) is a systemic inflammatory disease affecting muscles and several other organs, including gastrointestinal system. Dysphagia is the main symptom of gastrointestinal involvement, due to weakness of oropharyngeal and esophageal muscles; it can sometimes affect seriously the quality of life and can be refractory to traditional immunosuppressive and symptomatic treatments [1].
To date, few data are available to evaluate effectiveness and safety of Intravenous Immunoglobulins (IVIg) on esophageal involvement and multiple mechanisms of action have been proposed, such as interference with complement and membrane attack complex deposition, down regulation of intercellular adhesion molecule 1 in several cell types, down regulation of TGF $\beta 1$ expression in muscle fibers [2].

Here we describe a case of a woman presenting dysphagia related to polymyositis particularly complicated to manage.

\section{Case Report}

A 57-year-old woman, affected by polymyositis since 2003, was referred to our Rheumatology Unit for a severe dysphagia despite the ongoing therapy (low doses of steroids and Methotrexate [MTX] $7.5 \mathrm{mg}$ weekly).

At the onset, she presented severe muscle weakness, elevation of the Creatin-Kinase (CK) level (reaching 350 $\mathrm{IU} / \mathrm{L})$, Raynaud phenomenon, calcinosis, sicca syndrome, dysphagia, and gastric pyrosis, positivity for Antinuclear Antibodies (ANA) 1:160 speckled with no anti-Extractable Nuclear Antibodies (ENA). The patient underwent to a muscle biopsy showing changes in fibre size, myofiber degeneration and regeneration with diffuse or focal inflammatory infiltrates and a electromyography suggestive of myopathy. Finally, other connective tissue diseases, such as systemic sclerosis or mixed connective tissue disease were excluded; myositis-specific antibodies including anti-SRP were negative and the patient didn't take statins. Nail fold video capillaroscopy was negative for scleroder- 


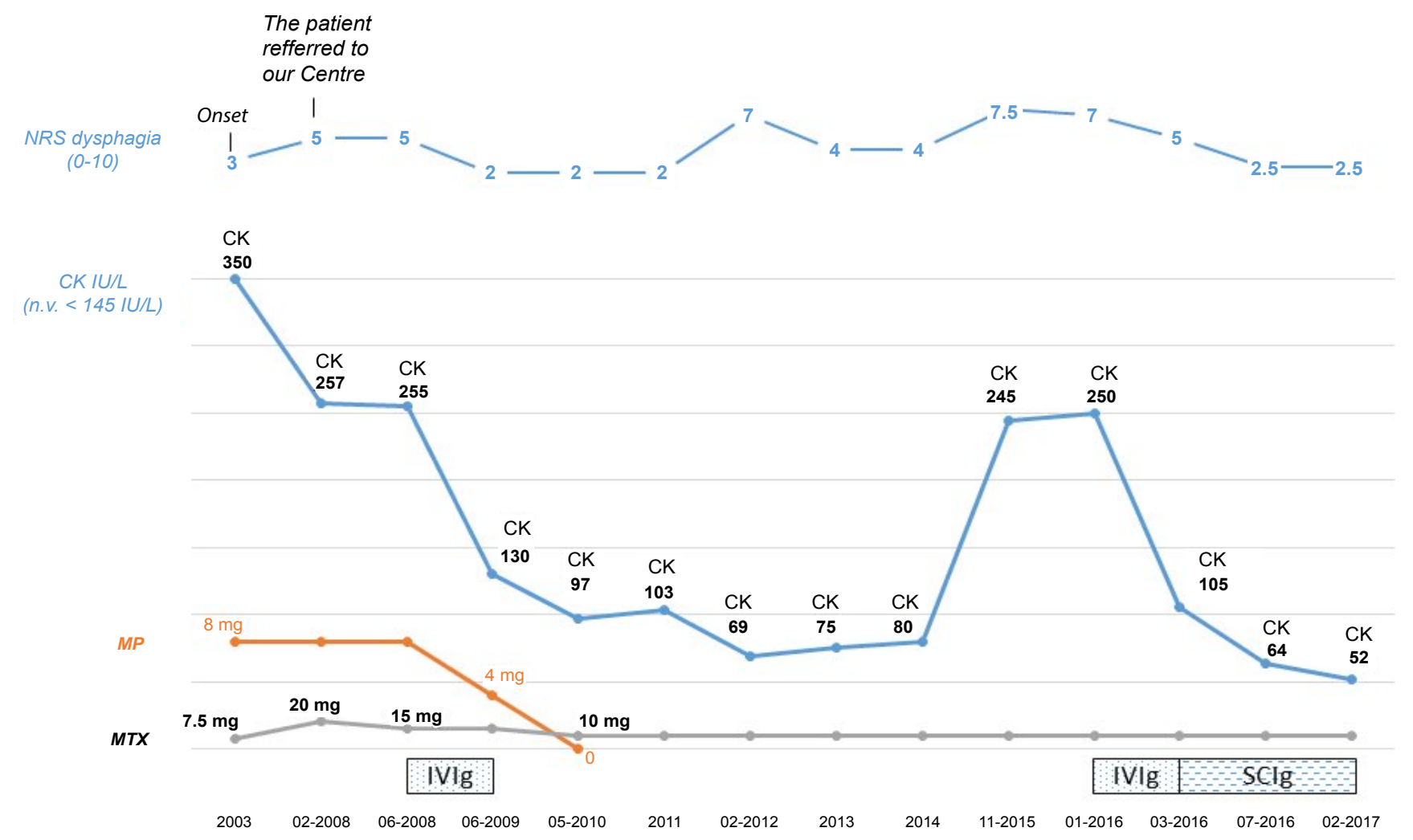

Figure 1: NRS: Number Rating; CK: Creatin-Kinase; IU/L: International Units per Liter; n.v.: normal value; MTX: Methotrexate; MP: Methylprednisone; IVIg: International Immunoglobulins; SCIg: Subcutaneous Immunoglobulins.

ma-like pattern [3], while a high-resolution chest tomography and the pulmonary function tests excluded an interstitial lung disease.

In 2008 she firstly referred to our Centre, because of a worsening of gastric pyrosis, dysphagia, and the appearance of dyspepsia, postprandial vomiting, constipation and progressive weight loss (5 kg lost in 10 months, reaching $45 \mathrm{~kg}$ ), with a Number Rating Scale (NRS) of gastrointestinal symptoms of 5/10.

At the admission, she showed a mild increase of $\mathrm{CK}$ $257 \mathrm{IU} / \mathrm{L}$ (normal value < 145), asthenia and muscle weakness. We increased MTX to $20 \mathrm{mg}$, but the patient showed a worsening in vomiting and nausea, probably due to the MTX itself. We associated only a low dosage of metylprednisone, since the patient already presented glucose intolerance and osteoporosis.

Therefore, to obtain a better disease control and a better compliance, we started monthly IVIg, $2 \mathrm{~g} / \mathrm{kg}$ in 3 days, associated to Methotrexate $15 \mathrm{mg}$ weekly and metylprednisone $8 \mathrm{mg}$ daily. The patient also maintained therapy with prokinetics and proton-pump inhibitors.

Her muscle strength, asthenia and dysphagia (NRS2/10) rapidly improved and CK levels progressively decreased, reaching normal values after 12 months (130 IU/L); IVIg was progressively discontinued and, after 2 years, we were able to reduce MTX dosage to $10 \mathrm{mg} /$ week and stop therapy with steroids. The clinical situation remained stable, with acceptable quality of life, for a long time (Figure 1).

In 2012, because of a worsening of the gastroenteric in- volvement (NRS 7/10), she underwent to esophageal manometry, and video fluoroscopy swallow study detecting esophageal acalasia, gastroesophageal reflux, esophagitis and motor-like dyspepsia gastroparesis. Prokinetic agents, Pyridostigmine, Trimebutine, $\mathrm{H} 2$ receptor antagonists or Proton-pump inhibitors were able to control the symptoms, without increasing MTX.

After 3 years of stable moderate dysphagia (NRS 4/10), in 2015 postprandial vomiting relapsed and dysphagia worsened (NRS 7, 5/10) again, and the patient became unable to assume solid food.

Monthly IVIg ( $2 \mathrm{~g} / \mathrm{kg}$ in 3 days) were started again, with a new quick improvement of dyspepsia, dysphagia and vomiting (NRS 5/10). After two months, Subcutaneous Immunoglobulins ( $\mathrm{SClg}$ ), at the dose of $10 \mathrm{~g}$ twice a week, were proposed to increase the compliance of the patient (difficulty to reach the hospital). After 4 months she reported a further and remarkable improvement of symptoms (NRS 2, 5/10) and quality of life. The condition remained stable and satisfying during the 6 additional month of follow-up.

\section{Discussion}

Dysphagia in the context of inflammatory muscle diseases, such as polymyositis or dermatomyositis and cancer associated myositis [4], is frequently described, with a highly variable prevalence of $30-60 \%[5,6]$.

Weakness of tongue, oropharyngeal and esophageal musculature, sometimes in the lower tract, can induce difficulties with swallowing. Patients report sensation of 
food sticking in the pharynx, coughing, choking, or regurgitation while eating, and difficulty with solid and dry foods. Dysphagia can sometimes be associated to several morbidities, including malnourishment, dehydration, social isolation, respiratory infections due to aspiration, and mortality [6].

Beyond symptomatic therapies, such as prokinetics, immunosuppressive therapy is the main treatment of PM related dysphagia. In particular, glucocorticoids are the mainstay of initial treatment of dysphagia. The addition of immunosuppressive drugs, such as methotrexate, azathioprine, mycophenolate mofetil and cyclosporine, is often required to control refractory disease, repeated disease flares, or to reduce the dose of corticosteroid therapy and associated side effects $[2,7]$.

Data from uncontrolled studies in PM, indeed, documented the benefit of IVIg: this treatment is considered a second line therapeutic option, especially in patients with disease refractory to glucocorticoids and/or immune suppressants. Moreover, IVIg seems to achieve disease control in patients with severe muscle weakness (i.e. dropped head syndrome) or dysphagia [2,8-11].

To date, few data are available to evaluate IVIg on esophageal involvement in refractory PM; usually the regimen applied is $2 \mathrm{~g} / \mathrm{kg}$ over 2-5 days. In 2002, Cherin, et al. carried out an open prospective study to evaluate the efficacy of IVIg in 35 subjects with PM that was refractory to traditional treatments. They administered IVIg in a regimen of $2 \mathrm{~g} / \mathrm{kg}$ over 2 days monthly for mean 4-6 months. Eleven subjects presented esophageal disorders (including dysphagia, regurgitation and gastroesophageal reflux) evaluated with clinical assessment and manometry. In 8/11 (73\%) patients they observed the complete disappearance of esophageal disorder [12].

In 2010, Marie, et al. retrospectively reviewed the medical records of 73 patients ( 39 with PM, 34 with DM) with steroid-resistant esophageal involvement, including dysphagia and gastroesophageal reflux. Patients were treated with IVlg ( $2 \mathrm{~g} / \mathrm{kg}$ over 2 days monthly for a mean of 7 months) and 60/73 (82\%) patients showed a rapid and significant improvement in esophageal symptoms [13].

In 2012, Miyasaka, et al. presented the results of a double-blind, placebo-controlled, crossover study designed to evaluate the efficacy and safety of polyethylene glycol-treated human IgG in 26 patients with corticosteroid-resistant PM (16 patients) or DM (10 patients). Nine patients presented dysphagia. They administered IVIg in a regimen of $2 \mathrm{~g} / \mathrm{kg}$ over 5 days. Among 7 patients with esophageal involvement in the treatment group, 5 showed a disappearance of the symptoms, while no changes were observed in the 2 remaining subjects in the placebo group [14].

SClg are a new therapeutic opportunity, which offers many advantages compared to IVIg, representing a safe alternative for difficult venous access, usually well accepted by patients $[2,15,16]$. SClg have been usefully employed in various immune-mediated disorders, such as chronic inflammatory demyelinating neuropathy, multifocal motor neuropathy, other than primary and acquired immunodeficiency $[15,17,18]$.

Pars K, et al. in 2013 reported a dramatic improve of dysphagia with IVIg in a 70-year-old patient affected by Inclusion Body Myositis (IBM). Then, the patient switched to $\mathrm{SClg}(0.77 \mathrm{~g} / \mathrm{kg} / \mathrm{month})$ and swallowing and body weight was constant over 4 years of follow up, preventing parental nutrition [19]. Also Cherin P, et al. reported 6 cases regarding patients affected by IBM describing a resolution of dysphagia with SClg treatment [20]. In a following study, the same authors described 19 patients affected by inflammatory myophaties, among which 10 affected by PM or $\mathrm{DM}$, successfully treated with $\mathrm{SClg}$, but no data were reported about dysphagia [21].

Danieli also described 11 patients affected by PM treated with $\mathrm{SClg}$ with different administration schedules, including 6 patients with dysphagia [22]. She reported an improvement of the clinical and laboratory features regarding weakness and CK levels in all patients, without describing the evolution of dysphagia [22,23].

Finally, in 2014, Cherin reported the case of a female patient, 49-years-old, presenting a refractory PM with increasing dysphagia, and subsequent significant weight loss. After the failure of steroids and immune suppressive drugs and after the discontinuation of IVIg therapy because of problematic venous access, SClg was given twice per week ( 2 then $1.3 \mathrm{~g} / \mathrm{kg} /$ month). Within 2 months, a progressive improvement in muscle strength and the normalization of serum CK were observed. Dysphagia was also resolved, preventing parenteral nutrition and allowing for normal daily activities [24].

Up to date, this is the second observation describing an improvement of dysphagia in a patient affected by PM treated with $\mathrm{SClg}$. This therapy has demonstrated to be safe, well accepted and this could be a reasonable therapeutic option also in patient with refractory dysphagia in the context of inflammatory muscle diseases, such as PM.

Further studies are needed to evaluate the effectiveness of SClg in the management of these patients.

\section{References}

1. Carstens PO, Schmidt J (2014) Diagnosis, pathogenesis and treatment of myositis: recent advances. Clin Exp Immunol 175: 349-358.

2. Anh-TuHoa S, Hudson M (2017) Critical review of the role of intravenous immunoglobulins in idiopathic inflammatory myopathies. Semin Arthritis Rheum 46: 488-508.

3. Manfredi A, Sebastiani M, Cassone G, Pipitone N, Giuggioli D, et al. (2015) Nailfold capillaroscopic changes in dermatomyositis and polymyositis. Clin Rheumatol 34: 279-284.

4. Neri R, Barsotti S, lacopetti V, lacopetti G, Pepe P, et al. (2014) Cancer-associated myositis: a 35-year retrospective study of a monocentric cohort. Rheumatol Int 34: 565-569. 
5. Mugii N, Hasegawa M, Matsushita T, Hamaguchi $Y$, Oohata S, et al. (2016) Oropharyngeal dysphagia in dermatomyositis: associations with clinical and laboratory features including autoantibodies. PLoS One 11: e0154746.

6. Mulcahy KP, Langdon PC, Mastaglia F (2012) Dysphagia in inflammatory myopathy: self-report, incidence, and prevalence. Dysphagia 27: 64-69.

7. Moghadam-Kia S, Aggarwal R, Oddis CV (2015) Treatment of inflammatory myopathy: emerging therapies and therapeutic targets. Expert Rev Clin Immunol 11: 1265-1275.

8. Foreman C, Russo P, Davies N, Hissaria P, Proudman S, et al. (2017) Use of intravenous immunoglobulin therapy for myositis: an audit in South Australian patients. Intern Med J 47: 112-115.

9. Oddis CV (2016) Update on the pharmacological treatment of adult myositis. J Intern Med 280: 63-74.

10. Lam CG, Manlhiot C, Pullenayegum EM, Feldman BM (2011) Efficacy of intravenous Ig therapy in juvenile dermatomyositis. Ann Rheum Dis 70: 2089-2094.

11. Huber AM, Giannini EH, Bowyer SL, Kim S, Lang B, et al. (2010) Protocols for the initial treatment of moderately severe juvenile dermatomyositis: results of a children's arthritis and rheumatology research alliance consensus conference. Arthritis Care Res (Hoboken) 62: 219-225.

12. Cherin $P$, Pelletier $S$, Teixeira $A$, Laforet $P$, Genereau $T$, et al. (2002) Results and long-term followup of intravenous immunoglobulin infusions in chronic, refractory polymyositis: an open study with thirty-five adult patients. Arthritis Rheum 46: 467-474.

13. Marie I, Menard JF, Hatron PY, Hachulla E, Mouthon L, et al. (2010) Intravenous immunoglobulins for steroid-refractory esophageal involvement related to 39 polymyositis and dermatomyositis: a series of 73 patients. Arthritis Care Res (Hoboken) 62: 1748-1755.

14. Miyasaka N, Hara M, Koike T, Saito E, Yamada M, et al (2012) Effects of intravenous immunoglobulin therapy in Japanese patients with polymyositis and dermatomyositis resistant to corticosteroids: a randomized double-blind placebo-controlled trial. Mod Rheumatol 22: 382-393.
15. Danieli MG, Gelardi C, Pedini V, Moretti R, Gabrielli A, et al. (2014) Subcutaneous IgG in immune-mediate diseases: proposed mechanisms of action and literature review. Autoimmun Rev 13: 1182-1188.

16. Hachulla E, Benveniste O, Hamidou M, Mouthon L, Schleinitz $\mathrm{N}$, et al. (2017) High dose subcutaneous immunoglobulin for idiopathic inflammatory myopathies and dysimmune peripheral chronic neuropathies treatment: observational study of quality of life and tolerance. Int J Neurosci 127: 516-523.

17. Haddad E, Barnes D, Kafal A (2012) Home therapy with subcutaneous immunoglobulins for patients with primary immunodeficiency diseases. Transfus Apher Sci 46: 315-321.

18. Compagno N, Cinetto F, Semenzato G, Agostini C (2014) Subcutaneous immunoglobulin in lymphoproliferative disorders and rituximab-related secondary Hypogammaglobulinemia: a single-center experience in 61 patients. Haematologica 99: 1101-1106.

19. Pars K, Garde N, Skripuletz T, Pul R, Dengler R, et al. (2013) Subcutaneous immunoglobulin treatment of inclusion-body myositis stabilizes dysphagia. Muscle Nerve 48: 838-839.

20. Cherin P, Delain JC, de Jaeger C, Crave JC (2015) Subcutaneous Immunoglobulin Use in Inclusion Body Myositis: A Review of 6 Cases. Case Rep Neurol 7: 227-232.

21. Cherin P, Belizna C, Cartry O, Lascu-Dubos G, de Jaeger C, et al. (2016) Long-term subcutaneous immunoglobulin use in inflammatory myopathies: A retrospective review of 19 cases. Autoimmun Rev 15: 281-286.

22. Danieli MG, Gelardi C, Pedini V, Logullo F, Gabrielli A, et al. (2017) Subcutaneous IgG in the myositis spectrum disorders. Curr Rheumatol Rev.

23. Danieli MG, Pettinari L, Moretti R, Logullo F, Gabrielli A, et al. (2011) Subcutaneous immunoglobulin in polymyositis and dermatomyositis: a novelapplication. Autoimmun Rev 10: 144149.

24. Cherin P, Delain JC, Crave JC, Cartry O (2015) Corrigendum to "high-dose subcutaneous immunoglobulins for the treatment of severe Treatment-resistant polymyositis". Case Rep Rheumatol 2015: 436736. 